\title{
On the maximum damping performance of piezoelectric switching techniques
}

Journal of Intelligent Material Systems and Structures

24(6) 717-728

(C) The Author(s) 2012

Reprints and permissions:

sagepub.co.uk/journalsPermissions.nav DOI: I0.1 I 77// 045389XI2445645 jim.sagepub.com (S)SAGE

\author{
Marcus Neubauer, Xu Han and Jörg Wallaschek
}

\begin{abstract}
Synchronized switch damping on inductor offers a high damping performance in a broad frequency range. It consists of an inductor and resistor in a serial configuration, which are connected and disconnected from the piezoceramics in an alternating manner by a switch. When the switch is triggered by the vibration itself, it adapts to different excitation frequencies especially in the low frequency range. This article presents a detailed study of the damping performance of the synchronized switch damping on inductor technique. Calculations are performed in a normalized way. The optimal tuning of synchronized switch damping on inductor network parameters is derived, and the corresponding maximum damping performance is obtained. The results are further compared to standard linear inductance-resistance networks. For a validation of the theoretical results, measurements on a clamped beam test rig are performed. Therefore, the synchronized switch damping on inductor circuit is realized as a synthetic impedance in a DSpace environment. The measurement results are in good agreement with the theoretical calculations.
\end{abstract}

\section{Keywords}

Control, piezoceramics, morphing, piezoelectric, autonomic structures

\section{Introduction}

Piezoceramics are widely used as actuators and sensors in technical systems. They offer very precise positioning and high dynamics, which makes them suitable for vibration damping especially in the high frequency range. An alternative to a fully active vibration control is piezoelectric shunt damping (Moheimani, 2003). It consists of an electrical network that is connected to the piezoceramics. The ceramics is embedded into the mechanical structure and couples the mechanical and the electrical systems by the piezoelectric effect. This energy conversion can be optimized by the placement of the piezoceramics within the mechanical structure. Detailed knowledge of the vibration modes of the structure is required for this.

The electrical network is designed with the aim to dissipate as much energy as possible, which generates a damping effect on the mechanical structure. The classical approach is to use a passive inductor-resistor network, which forms an electrical resonant circuit with the inherited capacitance of the piezoceramics (Hagood and Von Flotow, 1991; Tang and Wang, 2001). Tuning the electrical resonance frequency to the excitation frequency generates a resonance amplification which maximizes the voltage amplitudes and the dissipation within the resistance. These networks must be tuned to one specific frequency and are only effective in a narrow frequency range around the tuned frequency. However, there exist techniques for damping multiple modes simultaneously basically by adding additional inductor-resistor branches (Behrens and Moheimani, 2002; Fleming et al., 2003; Hollkamp, 1994; Wu, 1998).

In order to enhance the damping performance, active-passive hybrid piezoelectric networks (APPNs) are investigated (Tang and Wang, 2001). Such networks utilize the damping performance of passive networks and combine it with active elements like a negative capacitance (Neubauer et al., 2006) or negative resistors to enhance it.

Recently, various semiactive switching techniques have been studied in detail. A semiactive system is characterized by a passive system whose parameters can be online tuned during operation. A typical example for semiactive shunt damping techniques is switching

Institute of Dynamics and Vibration Research, Gottfried Wilhelm Leibniz University Hannover, Hannover, Germany

\section{Corresponding author:}

Marcus Neubauer, Institute of Dynamics and Vibration Research, Gottfried Wilhelm Leibniz University Hannover, Hannover 30167, Germany.

Email: neubauer@ids.uni-hannover.de 
networks. In these techniques, the electrical network is connected and disconnected by a switching device. Typically, the switching is triggered by the mechanical vibration itself, which makes these techniques adaptive to the excitation frequency. They are ideal for damping of mechanical systems with tonal vibrations (however, they are also capable to damp multiple frequencies, see Corr and Clark (2003), Clark and Schoenly (2005), Niederberger et al. (2004), and Neubauer et al. (2011)). Because of the adaption to the excitation frequency, they can be used in systems with time-varying vibration frequencies. Neubauer et al. (2006) presents the suppression of brake squealing of an automotive disk brake with a switching technique.

To the authors' best knowledge, the optimal tuning of synchronized switch damping on inductor (SSDI) networks for free vibrations have only been discussed in Ducarne et al. (2010), where for the first time the optimal tuning of the shunt parameters $L$ and $R$ have been proposed, together with the corresponding maximum damping. Their calculations are based on the transfer matrix of the system, which links the system state with the state one period time later.

This article presents a similar analysis, albeit with a different way of calculation. It is structured in the following way: first, the general description of a mechanical system with piezoelectric shunt damping is derived. Based on this, the energy dissipation for a displacement driven system is given. Further on, the damping of the free vibrations is studied. Optimal network parameters are obtained, which maximize the damping performance. Finally, the test rig and measurements are described. For the first time, the optimal tuning could be validated in measurements.

\section{Modeling of a mechanical oscillator with piezoceramics}

In Neubauer and Wallaschek (2010), the dynamics of mechanical systems with piezoceramics are derived based on a finite element description of the system followed by a modal reduction. The results are based on linearized equations of the mechanical system and piezoelectric effect. Further on, the system is reduced to one single mechanical mode only, cf. Figure 1 in order to get the most general results, yielding

$$
\left[\begin{array}{ll}
1 & 0 \\
0 & 0
\end{array}\right]\left[\begin{array}{l}
\ddot{x} \\
\ddot{q}
\end{array}\right]+\left[\begin{array}{cc}
\omega_{0}^{2}+\frac{\kappa^{2}}{C_{p}} & \frac{\kappa}{C_{p}} \\
\frac{\kappa}{C_{p}} & \frac{1}{C_{p}}
\end{array}\right]\left[\begin{array}{l}
x \\
q
\end{array}\right]=\left[\begin{array}{l}
0 \\
v
\end{array}\right]
$$

Herein, $x$ represents the modal vibration amplitude, $q$ and $v$ are the electrical charge and voltage at the electrodes, $\omega_{0}$ is the eigenfrequency of the system with short-circuit electrodes, $C_{p}$ the capacitance of the piezoceramics, and $\kappa$ the electromechanical coupling factor. The electromechanical coupling is determined by the
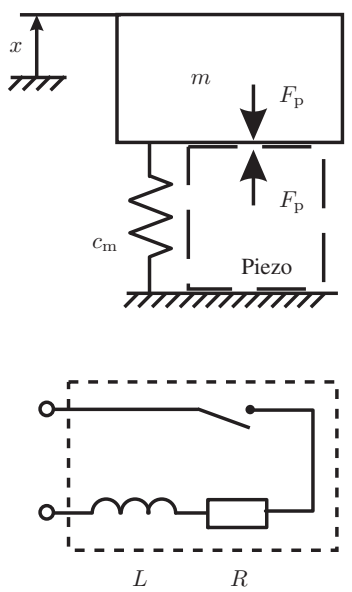

Figure I. One DOF oscillator with piezoceramics and the SSDI circuit.

DOF: degree of freedom; SSDI: synchronized switch damping on inductor.

piezoelectric properties as well as the mechanical coupling, which can be modified by the placement of the piezoceramics.

It is worthwhile to note that mechanical damping is neglected in this whole study. This is a typical assumption when piezoelectric shunt damping is discussed (Ducarne et al. (2010) is an exception here). But from the results found by these authors, the optimal tuning of the networks is not influenced strongly by the mechanical damping. Additionally, the lightweight structures, which are one application of the shunt damping technique, are usually very lightly damped.

When the switch is open, the piezoceramics is operated with isolated electrodes, which means no charge can flow. When the switch is closed, an inductanceresistance $(L R)$ network is connected to the electrodes of the piezoceramics, and the charge $q$ and voltage $v$ are related by the following differential equation

$$
L \ddot{q}+R \dot{q}+v=0
$$

Inserting this expression into equation (1) yields

$$
\left[\begin{array}{ll}
1 & 0 \\
0 & L
\end{array}\right]\left[\begin{array}{l}
\ddot{x} \\
\ddot{q}
\end{array}\right]+\left[\begin{array}{ll}
0 & 0 \\
0 & R
\end{array}\right]\left[\begin{array}{c}
\dot{x} \\
\dot{q}
\end{array}\right]+\left[\begin{array}{cc}
\omega_{0}^{2}+\frac{\kappa^{2}}{C_{p}} & \frac{\kappa}{C_{p}} \\
\frac{\kappa}{C_{p}} & \frac{1}{C_{p}}
\end{array}\right]\left[\begin{array}{l}
x \\
q
\end{array}\right]=\left[\begin{array}{l}
0 \\
0
\end{array}\right]
$$

In the following, two important cases will be discussed: In the first case, the system is excited by a harmonic displacement of the vibration mode under consideration and the corresponding damping performance is judged by the dissipated energy per vibration period. In the second case, the free vibrations are analyzed and the performance is judged by the reduction in amplitudes of the mechanical system. 


\section{Displacement driven system}

Let us now consider a harmonic displacement excitation of the mechanical system

$$
x(t)=\hat{x} \mathrm{e}^{j \Omega t}
$$

with amplitude $\hat{x}$ and frequency $\Omega$. We will analyze both the resonant $L R$ and the switching SSDI networks for this excitation.

\section{Resonant $L R$ shunting}

From the second line in equation (3), it follows

$$
L \ddot{q}+R \dot{q}+\frac{1}{C_{p}} q=-\frac{\kappa}{C_{p}} x
$$

Using the constitutive piezoelectric equations, the electrical charge can be substituted by the mechanical deformation and the electrical voltage

$$
q=\kappa x+C_{p} u_{p}
$$

Collecting excitation terms on the right side of the equation, we obtain

$$
L C_{p} \ddot{q}+R C_{p} \dot{u}_{p}+u_{p}=-\kappa(L \ddot{x}+R \dot{x})
$$

Subsequently, the stationary voltage amplitude for a harmonic excitation is obtained as follows

$$
\hat{u}_{p}=\frac{\kappa}{C_{p}} \frac{-L C_{p} \Omega^{2}+j R C_{p} \Omega}{-L C_{p} \Omega^{2}+j R C_{p} \Omega+1} \hat{x}
$$

In order to get generalized results, normalized parameters will be introduced

$$
\eta=\frac{\Omega}{\omega_{0, e l}}=\Omega \sqrt{C_{p} L}, \quad \zeta=\frac{R}{2} \sqrt{\frac{C_{p}}{L}}
$$

$\eta$ is the ratio between excitation frequency $\Omega$ and the electrical resonance frequency $\omega_{0, e l}$ and $\zeta$ is the normalized electrical damping ratio. With these parameters, the stationary voltage becomes

$$
\hat{u}_{p}=\frac{\kappa}{C_{p}} \frac{-\eta^{2}+2 \zeta \eta j}{1-\eta^{2}+2 \zeta \eta j} \hat{x}
$$

The amplitude is proportional to the piezoelectric coupling $\kappa$ and the vibration amplitude $\hat{x}$. Figure 2 shows a plot of the amplitude and the phase of the voltage versus frequency ratio $\eta$ for different damping ratios $\zeta$.

Additionally, the voltage amplitude is normalized to the amplitude with open electrodes. Obviously, the response strongly depends on the frequency. The maximum value is in good approximation located at resonance, $\eta=1$, and reads

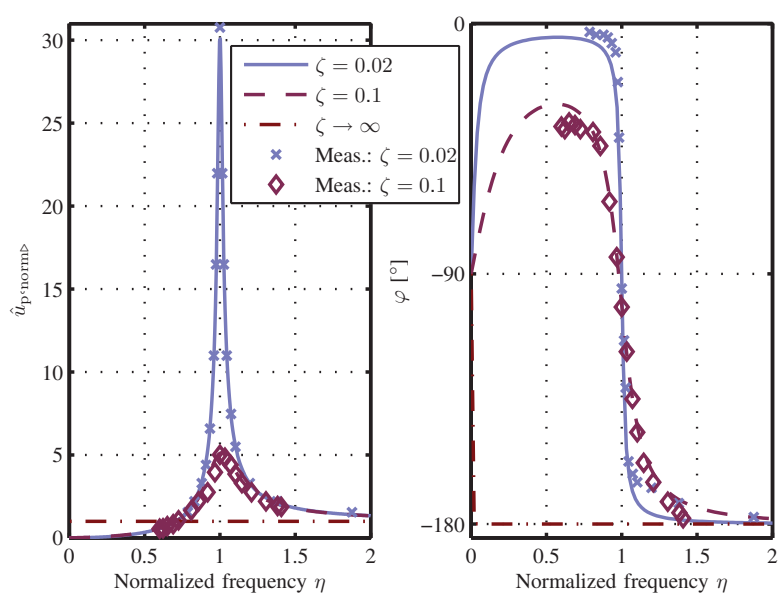

Figure 2. Stationary voltage amplitude and phase versus normalized frequency ratio $\eta$ for different electrical damping ratios $\zeta$.

$$
\hat{u}_{p, \text { norm. }}=\sqrt{1+\frac{1}{4 \zeta^{2}}} \approx \frac{1}{2 \zeta}
$$

The voltage amplitude is proportional to the inverse of the damping ratio $\zeta$. The phase between excitation and voltage crosses $90^{\circ}$ at $\eta=1$; therefore, also the energy dissipation is maximized. However, the narrow frequency range with high-voltage amplitudes requires precise tuning of the inductance. This circuit is therefore not suited for systems with time-varying frequencies or broadband excitation.

\section{SSDI technique}

The SSDI technique can be regarded as a combination of the resonant $L R$ shunting and a switch, which connects and disconnects the $L R$ shunt to the electrodes of the piezoceramics. The tuning of the SSDI shunt is totally different to the previous case. In contrast to the resonant $L R$ shunt, the SSDI is tuned to a rather high frequency, up to 1000 times higher than the excitation frequency (Lefeuvre et al., 2006). The switch is operated in such a way that it is closed at the moment of maximum deformation $\pm \hat{x}$ of the piezoceramics and is kept closed for precisely one half of the electrical period time, which is very short compared to the period of excitation. During this time, the charge at the electrodes is inverted. The remaining half of the mechanical period time, the switch is open and the charge is constant, until the switch is triggered by the next deformation maximum, and the whole process is repeated.

Mathematically, the SSDI technique is a piecewise linear system. For the (short) periods in which the switch is closed, the dynamics are the same as for the resonant $L R$ shunt given in equation (7). The case of open switch can be obtained by setting $R \rightarrow \infty$, which yields 

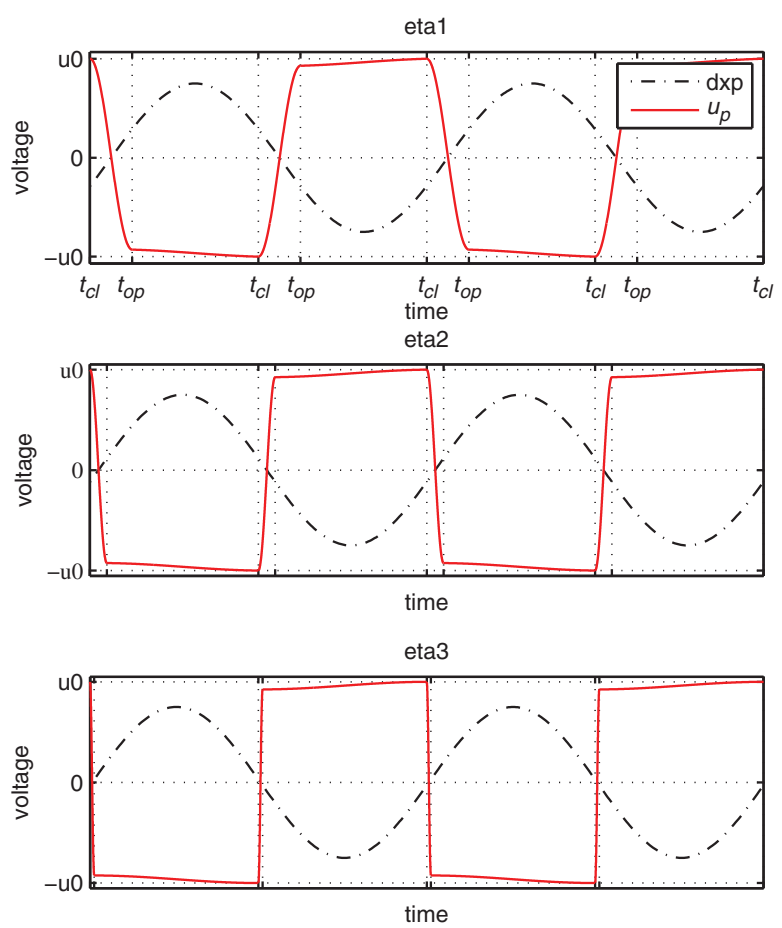

Figure 3. Timeplot of voltage signal with SSDI technique. SSDI: synchronized switch damping on inductor.

$$
u_{p}(t)=-\frac{\kappa}{C_{p}} x(t)+C
$$

During these times, the voltage changes proportionally with the deformation. Because of the voltage inversions, a constant offset $C$ is superimposed. This additional offset is generated by the voltage inversion in the SSDI technique and is responsible for the energy dissipation. Typical timeplots are given in Figure 3.

Each time a change in the switching state occurs, the final voltage value is taken as an initial condition for the subsequent time frame. The stationary voltage signal is then obtained by maintaining the stationarity condition $u_{p}(t)=u_{p}\left(t+T_{\text {excitation }}\right)$. This condition implies that the voltage is periodic with the period time of excitation $T_{\text {excitation }}$ so that it repeats after every period time $T$.

The SSDI technique adapts to different excitation frequencies, because the switching times are triggered by the vibration signal itself. As a result of this, the stationary voltage amplitude and the dissipated energy are nearly constant over a broad frequency range. Figure 4 presents the stationary voltage amplitudes $\hat{u}_{p, 0}$ for the SSDI and the resonant $L R$ shunt for two different damping values versus the frequency ratio $\eta$. For a frequency ratio of $\eta=1$, the excitation frequency equals the electrical resonance frequency, and the switch is always closed. Therefore, the SSDI behaves like the resonant $L R$ shunt in this case, and the stationary voltage amplitudes are the same. The voltage amplitudes and damping performance are slightly increased for

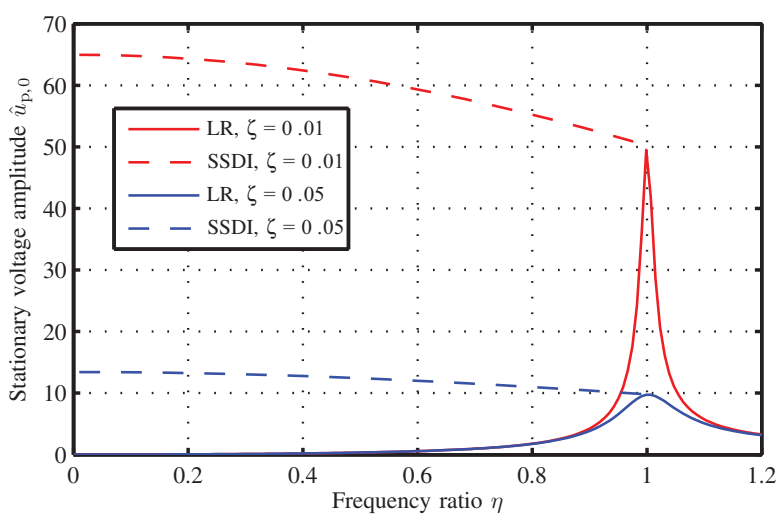

Figure 4. Stationary voltage amplitude of SSDI and resonant $L R$ shunts.

SSDI: synchronized switch damping on inductor; $L R$ : inductance-resistance.

lower excitation frequency (i.e. lower $\eta$ ). Also, in the range of $\eta>1$, the energy dissipation of SSDI equals the one of resonant $L R$ shunts. Here, the system does not adapt to the frequency anymore and the energy dissipation is drastically reduced. Practically, the SSDI circuit should be operated in a range $\eta \ll 1$, which means the electrical resonance frequency is much higher than the frequency of excitation.

With some reasonable approximations, cf. Neubauer and Wallaschek (2008), the stationary voltage amplitude $\hat{u}_{p, 0}$ can then be obtained as

$\hat{u}_{p, 0}=2 \frac{\kappa}{C_{p}} \frac{\cos \left(\frac{\pi \eta}{2 \nu}\right)+\left(1+e^{-\frac{\pi \zeta}{\nu}}\right) \cdot \sin \left(\frac{\pi \eta}{2 \nu}\right) \cdot \frac{\nu \eta}{\pi}}{1-e^{-\frac{\pi \zeta}{\nu}}} \hat{x}, \quad \nu=\sqrt{1-\zeta^{2}}$

The energy dissipation is strongly related to the hysteresis curves, as the enclosed area is proportional to the dissipation. These hysteresis curves of the SSDI techniques are given in Figure 5. The voltage signal $u_{p}(t)$ is

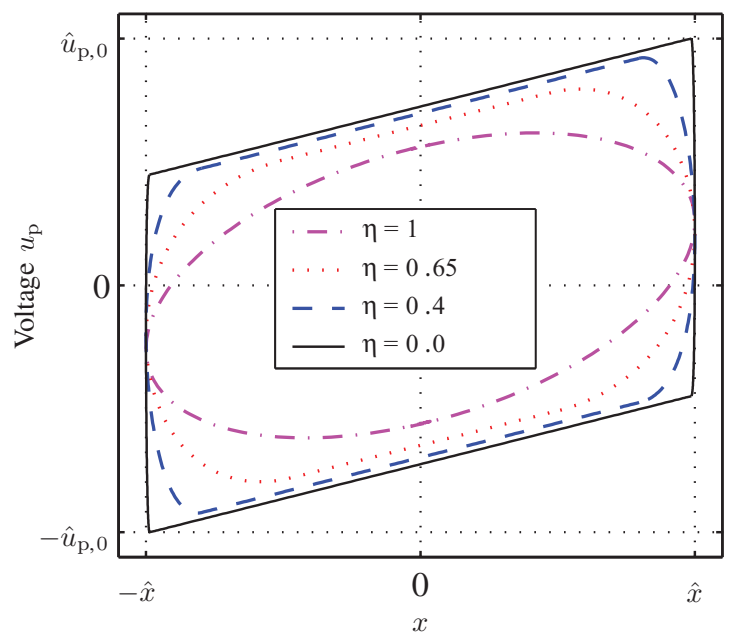

Figure 5. Hysteresis curves for SSDI technique and different frequency ratios $\eta$.

SSDI: synchronized switch damping on inductor. 
given versus the deformation $x(t)$. For all frequency ratios, the periodic response results in closed loops.

In Figure 5, two border cases can be distinguished: For $\eta=0$, the voltage inversion occurs instantaneously. The switch is opened for the whole half excitation period and only opened for one time instant. The corresponding hysteresis curve is a parallelogram where the gradient depends on the piezoelectric coupling $\kappa$. The other extremum is represented by $\eta=1$. In this case, the electrical resonant frequency equals the excitation frequency, and therefore, the switch would always be closed. The SSDI degenerates to the linear $L R$ shunt. The corresponding hysteresis curve is elliptic.

The dissipated energy is proportional to the enclosed area. It can already be concluded that the energy of SSDI is higher because of the following two facts.

(1) The stationary voltage amplitude $\hat{u}_{p, 0}$ is higher than that for resonant $L R$.

(2) The voltage is kept for a longer time at a high level, especially for fast voltage inversions.

Mathematically, the area $A$ of the hysteresis curves can be divided into the parallelogram area and two halves of an ellipse to the left and to the right, during which the voltage is inverted

$$
E_{\text {diss }}=2 \kappa x^{*}\left(\hat{u}_{p, 0}+u_{p, \text { after }}\right)+\pi \frac{\hat{u}_{p, 0}+u_{p, a f t e r}}{2}\left(\hat{x}-x^{*}\right)
$$

$x^{*}$ corresponds to the vibration amplitude at the time the switch is closed. This value depends on the frequency ratio $\eta$

$$
x^{*}=\hat{x} \cos \left(\frac{\eta}{2} \pi\right)
$$

The faster the inversion, the higher the amplitude $x^{*}$. For an instantaneous inversion $(\eta=0)$, it appears exactly at the maximum amplitude, $x^{*}=\hat{x}$. The voltage $u_{p, \text { after }}$ directly after inversion reads

$$
u_{p, a f t e r}=\hat{u}_{p, 0}-\Delta u_{p}, \quad \Delta u_{p}=2 \frac{\kappa}{C_{p}} \cos \frac{\pi \eta}{2 \nu} \hat{x}
$$

The stationary case, which is discussed here, requires that the change in voltage $\Delta u_{p}$ during the periods with closed switch equals the change in voltage during open switch.

Finally, the dissipated energy is obtained as a function of the frequency ratio $\eta$ and damping ratio $\zeta$, cf. Figure 6 . For the stationary voltage amplitude, the approximate solution according to equation (13) together with equation (13) is taken. This result is in very good agreement with the precise solution for lowfrequency ratios $\eta$ (which is the typical range for SSDI technique anyways), but differs from the correct results for higher values of $\eta$. Therefore, the results for SSDI

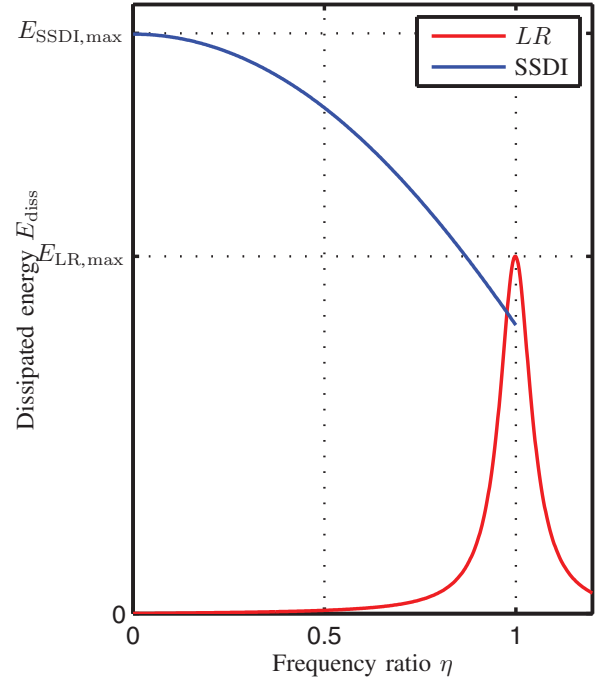

Figure 6. Dissipated energy versus frequency ratio $\eta$ for SSDI and resonant $L R$ shunts.

$S S D I$ : synchronized switch damping on inductor; $L R$ : inductance-resistance.

and $L R$ do not agree for $\eta=1$ although they theoretically should do so.

Generally, it can be concluded that the dissipated energy for the SSDI technique is higher than the resonant $L R$ shunt, and it decreases with the increasing frequency ratio $\eta$. The maximum energy dissipation for the SSDI technique appears at $\eta=0$ and for the linear $L R$ shunt approximately at $\eta=1$

$$
\begin{gathered}
E_{S S D I, \max }=4 \frac{\kappa^{2}}{C_{p}} \frac{1+\mathrm{e}^{-\pi \zeta}}{1-\mathrm{e}^{-\pi \zeta}} \hat{x}^{2} \\
E_{L R, \max }=\frac{\kappa^{2}}{C_{p}} \frac{1}{2 \zeta} \pi \hat{x}^{2}
\end{gathered}
$$

Both results have the same dependency with the vibration amplitude $\hat{x}$ and piezoelectric coupling $\kappa$. For small electrical damping ratios $\zeta$, which is the preferred case, the ratio between the dissipated energies can be calculated as

$$
r_{E}=\frac{E_{S S D I, \max }}{E_{L R, \max }}=\frac{16}{\pi^{2}} \approx 1.62
$$

The SSDI technique therefore - assuming an instantaneous voltage inversion with $\eta=0$-offers an energy dissipation that is about $62 \%$ higher than an $L R$ shunt driven in resonance. Moreover, the SSDI technique has a high energy dissipation over a broad frequency range, while the resonant $L R$ shunt reaches these levels only around the resonance frequency.

\section{Free vibrations}

Second, the case of free vibrations will be discussed. Basis for the following calculations is equation (3). For the further analysis, the inductance $L$ is normalized by 
an additional frequency ratio $\eta_{e l}$, while the resistance is again described by the damping ratio $\zeta$. Additionally, eigentimes $\tau$ and $\tau_{e l}$ are defined

$$
\begin{aligned}
& \omega_{i s o}^{2}=\omega_{0}^{2}+\frac{\kappa^{2}}{C_{p}}, \quad \omega_{e l}^{2}=\frac{1}{L C_{p}} \\
& K^{2}=\frac{\kappa^{2}}{C_{p} \omega_{0}^{2}+\kappa^{2}}, \quad \eta_{e l}=\frac{\omega_{e l}}{\omega_{i s o}}=\sqrt{\frac{1}{L\left(\omega_{0}^{2} C_{p}+\kappa^{2}\right)}} \\
& \tau=\omega_{\text {iso }} t, \quad \tau_{e l}=\omega_{e l} t, \quad(\bullet)=\omega_{\text {iso }}()^{\prime}
\end{aligned}
$$

This time, the normalized frequency ratio does not describe the ratio of excitation frequency and electrical resonant frequency, but the ratio between mechanical and electrical resonant frequencies. The generalized electromechanical coupling coefficient $K$ is strongly related to the material coupling $\kappa$, but includes additional parameters of the mechanical system. This parameter describes how much the mechanical vibration mode can be influenced by the piezoceramics.

After some mathematical conversions, the equations can be rewritten as

$$
\left[\begin{array}{cc}
1 & 0 \\
0 & \frac{1}{\eta_{e l}^{2}}
\end{array}\right]\left[\begin{array}{c}
x^{\prime \prime} \\
\frac{q^{\prime \prime}}{\kappa}
\end{array}\right]+2 \frac{\zeta}{\eta_{e l}}\left[\begin{array}{ll}
0 & 0 \\
0 & 1
\end{array}\right]\left[\begin{array}{c}
x^{\prime} \\
\frac{q^{\prime}}{\kappa}
\end{array}\right]+\left[\begin{array}{cc}
1 & K^{2} \\
1 & 1
\end{array}\right]\left[\begin{array}{l}
x \\
\frac{q}{\kappa}
\end{array}\right]=\left[\begin{array}{l}
0 \\
0
\end{array}\right]
$$

The derivative in time $t$ is described by $(\bullet)$ while the derivative in eigentime $\tau$ by ()$^{\prime}$.In this normalized form, the dynamics of the system is described by only three parameters, which are the frequency ratio $\eta_{e l}$, the electrical damping $\zeta$, and the generalized coupling coefficient $K$.

Like in the previous section, this system is piecewise linear, and the resistance value changes with the operation of the switch.

\section{Dynamics of the system with SSDI technique}

The system with piezoelectric SSDI network is a piecewise linear system during the times the switch is open or closed. However, as the time of the closed switch is only a small fraction of the overall time, this is often approximated by an instantaneous charge inversion. The optimization of such a system can be done using the transform matrix, which links the system state with the state one period time later, cf. Ducarne et al. (2010). In the following, a different way of optimizing the network parameters is described, which is related to the transfer matrix description. The time signals of the electrical charge and the mechanical vibration amplitude are calculated for one half period. The reduction of mechanical and electrical amplitudes during this half period are then related by a stationarity condition, and finally the corresponding mechanical damping ratio $D$ is obtained as a function of the electrical damping ratio $\zeta$.
The half period under investigation starts with open electrodes and arbitrary initial conditions $x_{p, 0}=$ $x(\tau=0), \dot{x}_{0}=0, q_{0}=q(\tau=0)$, which are the vibration amplitude, the velocity, and electrical charge at the beginning. As the charge $q$ is constant for open switch, equation (21) can be rewritten as

$$
x^{\prime \prime}(\tau)+x(\tau)=-\frac{K^{2}}{\kappa} q_{0}
$$

The general solution for this step function reads

$$
x(\tau)=x_{p, 0} \cos \tau-\frac{K^{2}}{\kappa} q_{0}(1-\cos \tau)
$$

and the final value after one half period $\tau=\pi$, termed $x^{*}$, is obtained as

$$
x^{*}=x(\tau=\pi)=-x_{p, 0}-2 \frac{K^{2}}{\kappa} q_{0}
$$

The electrical charge $q$ on the other hand stays constant at $q_{0}$ during the time with open switch and only changes during the inversion when the switch is closed.

The period with closed switch can again be described by equation (21). Here, the initial conditions are the final values of the previous time frame, $x_{p, 0, i+1}=x^{*}=-x_{p, 0, i}-2 \frac{K^{2}}{\kappa} q_{0}$ and $q=q_{0}$ (indices $i$ and $i+1$ refer to the $i$ th and $i+1$ th periods, respectively. These indices are omitted on the following for a better readability). Described in its electrical eigentime $\tau_{e l}$, the differential equation according to equation (21) for closed switch reads

$$
q^{\prime \prime}\left(\tau_{e l}\right)+2 \zeta q^{\prime}\left(\tau_{e l}\right)+q\left(\tau_{e l}\right)=-\kappa x^{*}
$$

As the electrical inversion time is very fast and occurs during the deformation maxima, the deformation $x^{*}$ can be assumed to be constant during this time. Then, the charge $q$ changes according to the step response

$$
\begin{aligned}
& q\left(\tau_{e l}\right)=\mathrm{e}^{-\zeta \tau_{e l}}\left[\cos \nu \tau_{e l}+\frac{\zeta}{\nu} \sin \nu \tau_{e l}\right] q_{0} \\
& -\kappa x^{*}\left[1-\mathrm{e}^{-\zeta \tau_{e l}}\left(\cos \nu \tau_{e l}+\frac{\zeta}{\nu} \sin \nu \tau_{e l}\right)\right], \quad \nu=\sqrt{1-\zeta^{2}}
\end{aligned}
$$

The switch is supposed to be opened at the time the charge is inverted, which occurs at $\tau_{e l}=\pi / \nu$ with a final charge $q^{*}$ of

$$
q^{*}=q\left(\tau_{e l}=\pi / \nu\right)=-\mathrm{e}^{-\pi \zeta}\left(\kappa x^{*}+q_{0}\right)-\kappa x^{*}
$$

with $\nu=\sqrt{1-\zeta^{2}} \approx 1$ for small electrical damping. Inserting the mechanical amplitude $x^{*}$ from equation (24) yields

$$
q^{*}=-e^{-\pi \zeta} q_{0}+\kappa\left(1+e^{-\pi \zeta}\right)\left(x_{p, 0}+2 \frac{K^{2}}{\kappa} q_{0}\right)
$$




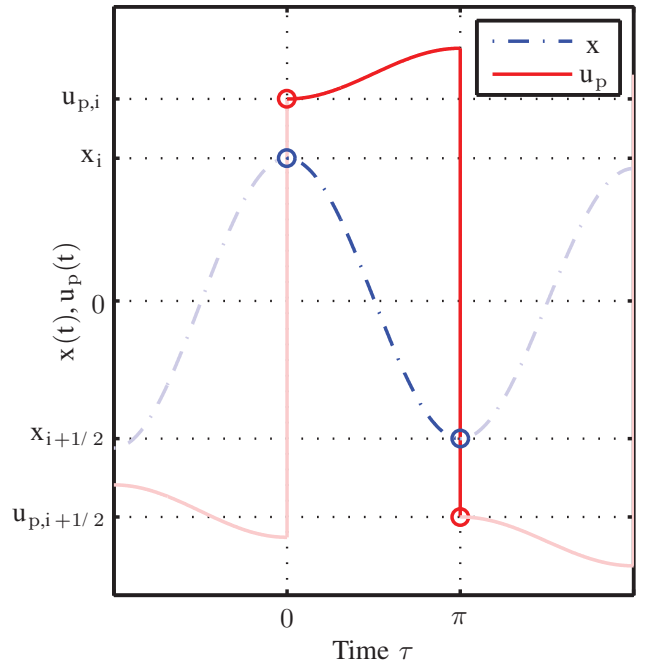

Figure 7. Time signals of $x$ and $u_{\mathrm{p}}$.

The following half periods could be obtained by the same calculations by taking the final values $x^{*}, q^{*}$ as the new initial conditions $x_{p, 0}, q_{0}$. Figure 7 shows an exemplary plot of the time signals for $x$ and $u_{p}$. Instead of the charge $q$, the electrical voltage $u_{p}$ is given, which would be easier to measure. Even though the charge is constant during each half vibration period, the electrical voltage slightly changes due to the mechanical vibration amplitudes. A slight reduction in mechanical amplitude due to the energy dissipation can be observed in the timeplots.

\section{Determination of the mechanical damping}

In order to describe the evolution of the vibrations, it is useful to introduce two new parameters, which are the reduction in mechanical amplitude $k_{x}$ and in electrical charge $k_{q}$ during each half period

$$
\begin{gathered}
k_{x}=\left|\frac{x_{p, 0}}{x^{*}}\right|=\frac{1}{1+2 \frac{K^{2}}{\kappa} \frac{q_{0}}{x_{p, 0}}} \\
k_{q}=\left|\frac{q_{0}}{q^{*}}\right|=\frac{q_{0}}{e^{-\pi \zeta} q_{0}-\left(1+e^{-\pi \zeta}\right) \kappa\left(x_{p, 0}+2 \frac{K^{2}}{\kappa} q_{0}\right)}
\end{gathered}
$$

Due to the inversion in sign, the absolute value is taken. These reduction parameters do not depend on the amplitude values itself; therefore, without loss in generality, we can define $x_{p, 0}=1$ as the initial mechanical vibration amplitude.

In general, the reduction in mechanical amplitude $k_{x}$ and in electrical amplitude $k_{q}$ can be different. This would mean that the mechanical and electrical amplitudes do not follow the same trend. The result of this would be a "pulsing" phenomenon, already observed in Ducarne et al. (2010). They show that the corresponding damping performance is lower in this case.
For higher electrical damping $\zeta$, the mechanical and electrical signals reduce in an identical trend. This fact can be used to formulate a mathematical criterion for optimal network parameters, which are the lowest $\zeta$ values for which the pulsing effect does not occur. Therefore, the quasi-stationary condition now requires that $k_{x}$ equals $k_{q}$. This yields a quadratic equation for the unknown charge $q_{0}$

$$
\frac{K^{2}}{\kappa} q_{0}^{2}-\frac{e^{-\pi \zeta}-1-2 K^{2}\left(1+e^{-\pi \zeta}\right)}{2} q_{0}+\kappa \frac{1+e^{-\pi \zeta}}{2}=0
$$

which has the solutions

$$
\begin{gathered}
\frac{K^{2}}{\kappa} q_{0}=-\frac{1+2 K^{2}\left(1+e^{-\pi \zeta}\right)-e^{-\pi \zeta} \pm \sqrt{d}}{4}, \\
d=\left[1+2 K^{2}\left(1+e^{-\pi \zeta}\right)-e^{-\pi \zeta}\right]^{2}-8\left(1+e^{-\pi \zeta}\right) K^{2}
\end{gathered}
$$

The result of this equation is the electrical charge $q_{0}$ for a mechanical vibration amplitude of $x_{p, 0}=1$. This value depends on the generalized coupling coefficient $K$ and the electrical damping ratio $\zeta$. From the two solutions $\pm \sqrt{d}$, only the solution with $+\sqrt{d}$ makes sense. With the term $\frac{K^{2}}{\kappa} q_{0}$ from equation (32), the reduction in vibration amplitude $k_{x}$ can readily be found when inserting the result into equation (29). From this reduction in amplitude during the free vibrations, the resulting mechanical damping ratio $D$ is as follows

$$
D=\frac{2 \ln \left|\frac{x_{p, 0}}{x^{*}}\right|}{2 \pi}=\frac{\ln \left(k_{x}\right)}{\pi}
$$

and inserting the previous results, it is finally calculated as

$$
D=\frac{\ln \left(\frac{2}{\left(1-2 K^{2}\right)\left(1+e^{-\pi \zeta}\right)+\sqrt{d}}\right)}{\pi}
$$

This general result shows the mechanical damping ratio as a function of the generalized coupling coefficient $K$ as well as the electrical damping ratio $\zeta$ (also the discriminant $d$ is a function of $K$ and $\zeta$ only). This function is drawn in Figure 8. Depending on the discriminant $d$, the solution is either a real value or complex. In any case, the damping of the system is described by the real value of the result, which is projected in Figure 8. For typical system parameters, where the electrical damping is higher than a certain value $\zeta_{\text {opt }}$, the result is real. These cases belong to the monotonic decreasing vibration amplitudes. For one certain electrical damping ratio $\zeta=\zeta_{\text {opt }}$, the discriminant is zero, $d=0$ and one real solution exists. For smaller electrical damping ratios $\zeta<\zeta_{\text {opt }}$, the discriminant is negative, and two complex conjugate solutions exist. The corresponding time signals of mechanical vibration and electrical charge show a distinct low-frequency oscillation of the energy between mechanical and electrical during the 


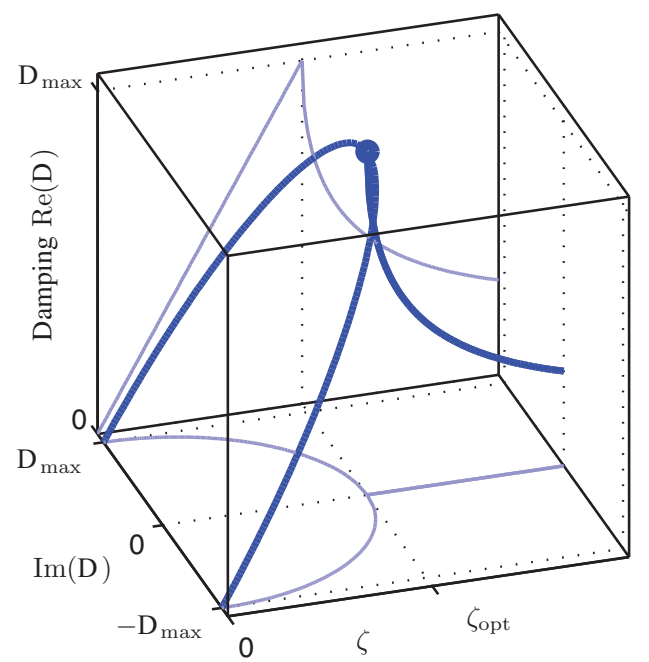

Figure 8. Resulting damping $D$ versus electrical damping ratio $\zeta$.

free vibrations. The damping $D$ corresponds to the real part $\operatorname{Re}(D)$ of the complex solution, while the imaginary part $\operatorname{Im}(D)$ describes the frequency of the energy oscillation.

In the range $0<\zeta<\zeta$ opt , the damping increases nearly linearly with $\zeta$. For $\zeta=0$ also the damping is zero, as the overall system is conservative then (notice that mechanical damping is neglected in the calculations). Above $\zeta_{\text {opt }}$ the damping $\operatorname{Re}(D)$ sharply reduces first, which means the system is very sensitive for small changes in $\zeta$.

The projection of the imaginary part $\operatorname{Im}(D)$ depicts a circle when the axes are scaled accordingly. The imaginary part, which is the frequency of the energy pulsation between mechanical and electrical subsystems, sharply increases from zero at $\zeta_{\text {opt }}$ and interestingly reaches $D_{\max }$ for $\zeta=0$.

The different time signals of the free vibrations are depicted in Figure 9. Beside the mechanical amplitude $x$ and the electrical charge $q$, the mechanical and electrical energies of the system are shown. They are calculated as

$$
\begin{aligned}
& E_{\text {mech }}=\frac{1}{2} c x^{2} \\
& E_{\text {elec }}=\frac{1}{2} C_{p} u_{p}^{2}
\end{aligned}
$$

For high electrical damping $\zeta>\zeta_{\text {opt }}$, the mechanical and electrical amplitudes and energies all reduce monotonically, and after a short initial phase, the amplitude
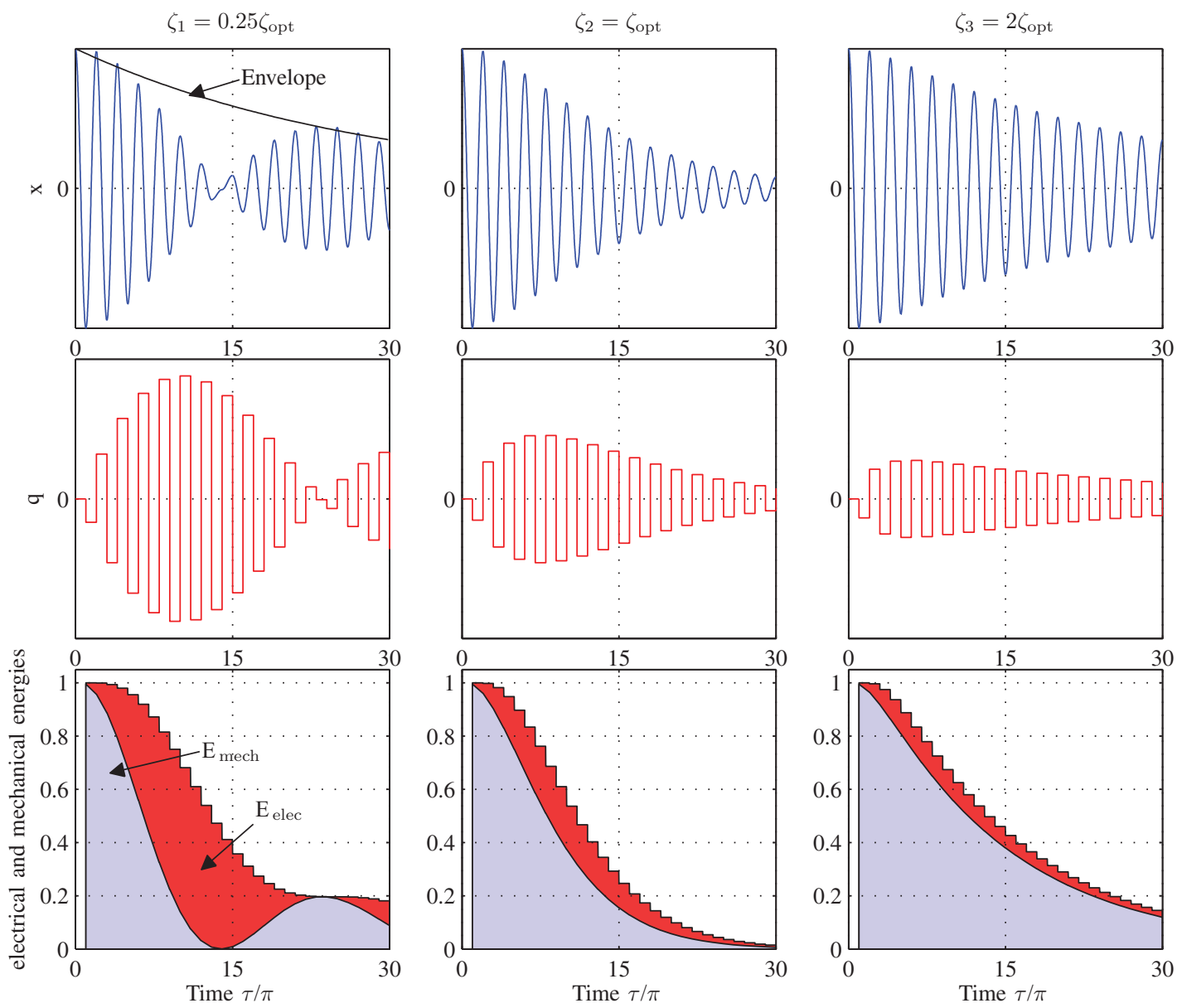

Figure 9. Time signals of $x, q$, and mechanical and electrical energies of the system. 
ratio is constant. The same is true for the optimal electrical damping $\zeta_{\text {opt }}$. In this case, the portion of electrical energy is higher, and the damping performance is maximized. In these cases, the force $F_{p}$ generated by the piezoceramics acts always against the deformation velocity $\dot{x}$. This can be seen in Figures 7 and 9 .

But if the electrical damping is lower than the optimal value, $\zeta<\zeta_{\text {opt }}$, a pulsing between mechanical and electrical energy occurs. In the beginning, the mechanical damping is mainly transformed to electrical energy. This leads to a fast reduction of the mechanical vibration amplitudes. But only a small part of the energy is dissipated, the main part is only converted into electrical energy. As a result, the electrical energy in the system increases, and at a certain time, the overall energy is purely electrical. The mechanical system has come to a rest, but the piezoceramics stores electrical energy at that moment. In the following, this energy is transferred back to mechanical energy, so that the system is excited to mechanical vibrations. The sign of the electric charge compared to the deformation velocity $\dot{x}$ has changed, so that the energy flows from electrical to mechanical subsystem.

Simultaneous to this repeating pulsation, the overall amplitudes are reduced due to the energy dissipation. However, the reduction is less than for the optimal case $\zeta_{\text {opt }}$.

\section{Determination of the optimal electrical damping $\zeta_{\text {opt }}$ and maximum damping $D_{\max }$}

With the results derived above, it is now possible to determine the optimal electrical damping ratio $\zeta_{o p t}$, which results in the maximum mechanical damping $D$. Based on the results of Figure 8, the maximum damping can be expected at that specific value $\zeta$, at which the result $D$ changes from purely real to complex. Therefore, the optimal $\zeta$ can be obtained by setting the discriminant $d$ equal to zero. Solving the equation for $d$ results in

$$
\zeta_{\text {opt }}=\frac{\ln \left(\frac{1-4 K^{2}+4 K^{4}}{1-4 K \sqrt{1-K^{2}}+4 K^{2}-4 K^{4}}\right)}{\pi}
$$

The term can be inserted into equation (34) for obtaining the corresponding maximum damping $D_{\max }$

$$
D_{\max }=\frac{\ln \left(\frac{1-2 K^{2}}{1-2 K \sqrt{1-K^{2}}}\right)}{\pi}
$$

Both results are only functions of the generalized coupling coefficient $K$ of the system. In Figure 10, they are drawn versus the coupling coefficient $K$. The optimal electrical damping ratio is normalized to $K$. For both results, approximations can be given

$$
\zeta_{\text {opt }} \approx \frac{4}{\pi} K, \quad D_{\max } \approx \frac{2}{\pi} K
$$
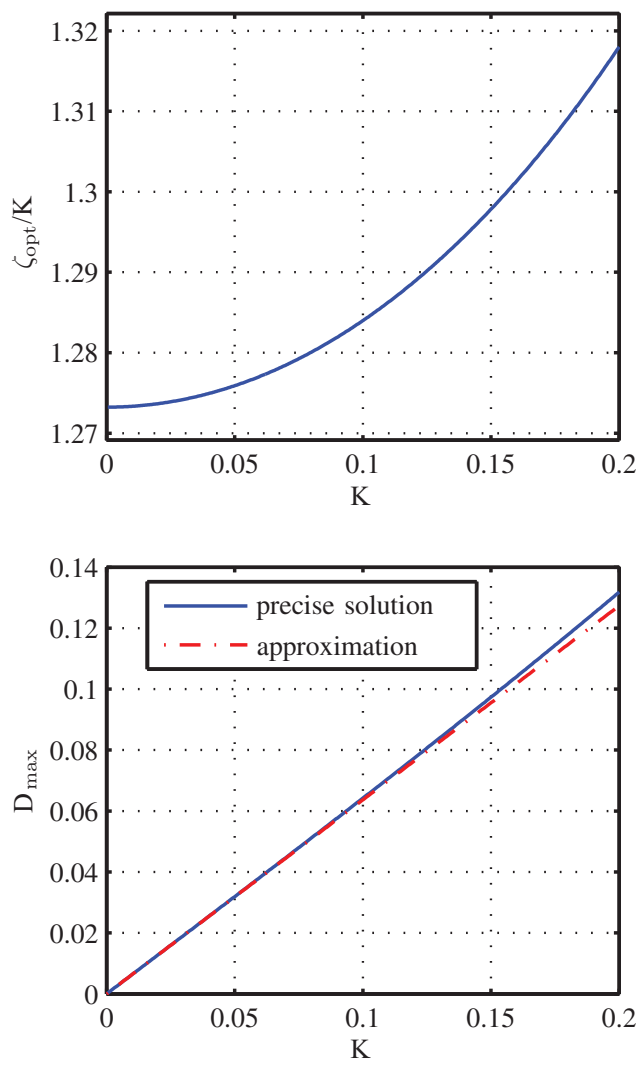

Figure 10. Optimal electrical damping ratio $\zeta_{\text {opt }}$ and resulting maximum damping $D_{\max }$ versus generalized coupling coefficient $K$.

which are exact when $K$ approaches zero, $K \rightarrow 0$.

These values can be used to compare the damping performance of the SSDI technique with the resonant LR shunting. According to Preumont (2006), the optimal electrical damping ratio and the corresponding damping are

$$
\left.\zeta_{\text {opt }, L R} \approx K, \quad D_{\max , L R} \approx \frac{K}{2}\right]
$$

These results show a remarkable analogy to the new found expressions for the SSDI technique: in both cases, the optimal electrical damping $\zeta_{\text {opt }}$ and the resulting maximum damping $D_{\max }$ depend linearly on $K$. Also, in both cases, $D_{\max }$ is half of the optimal electrical damping ratio $\zeta_{\text {opt }}$. The performance of the SSDI is, however, higher

$$
\frac{D_{\max , S S D I}}{D_{\max , L R}}=\frac{4}{\pi}=1.27
$$

Practically, it might be difficult to realize the optimum electrical damping value $\zeta_{\text {opt }}$. Due to the losses in the switching circuit, the typical damping values are in the range of $\zeta=0.1$ (Richard et al., 2000). The generalized coupling coefficient and therefore the optimum $\zeta$, on the other hand, are in many cases smaller. 
Table I. Beam and piezoceramics dimensions and material parameters.

\begin{tabular}{llll}
\hline Parameter & Beam & Piezo I & Piezo 2 \\
\hline Width & $30 \cdot 10^{-3} \mathrm{~m}$ & $20 \cdot 10^{-3} \mathrm{~m}$ & $9 \cdot 10^{-3} \mathrm{~m}$ \\
Length & $400 \cdot 10^{-3} \mathrm{~m}$ & $65 \cdot 10^{-3} \mathrm{~m}$ & $30 \cdot 10^{-3} \mathrm{~m}$ \\
Thickness & $1 \cdot 10^{-3} \mathrm{~m}$ & \\
Young's modulus & $190 \cdot 10^{9} \mathrm{~N} / \mathrm{m}^{2}$ & \\
Density & $7850 \mathrm{~kg} / \mathrm{m}^{3}$ & \\
$f_{\mathrm{l}, \text { iso }}$ & $5.8289 \mathrm{~Hz}$ & & \\
$f_{\mathrm{l} \text {, short }}$ & $5.8158 \mathrm{~Hz}$ & & $130 \cdot 10^{-3} \mathrm{~m}$ \\
$K$ & 0.067 & $10 \cdot 10^{-3} \mathrm{~m}$ & $-180 \cdot 10^{-12} \mathrm{pC} / \mathrm{N}$ \\
Location from clamped end & & $-180 \cdot 10^{-12} \mathrm{pC} / \mathrm{N}$ & $47 \mathrm{nF}$ \\
Piezoelectric constant $d_{31}$ & & $87 \mathrm{nF}$ & \\
Capacitance & & & \\
\hline
\end{tabular}

Additionally, it must be noted that these results are theoretical values which are valid for an instantaneous voltage inversion.

\section{Measurements}

In order to validate the theoretical results, a test rig has been set up. It consists of a clamped beam with attached piezoceramics for vibration damping. One piezo of type MFC M8528-P2 is connected with the shunted circuit used for damping, and the second piezo of type MFC M2814-P2 is used as a sensor. As the optimal tuning of the network parameter depends on the generalized coupling coefficient $K$, the eigenfrequencies of the beam are measured for the cases of isolated electrodes and shortcircuit electrodes of the piezo used for damping. The properties and measured frequencies of the test rig are summarized in Table 1.

It is the intention that the measurements agree with the assumptions of the mathematical model. Therefore, a synthetic impedance is used for the measurements. The design is similar to the one proposed in Fleming et al. (2000) and Fleming and Moheimani (2006). The metal-oxide semiconductor field-effect transistors (MOSFETs) that are used in the analog SSDI boards (cf. Niederberger and Morari, 2006), operate like a resistor, controlled by the gate voltage relative to both the source and drain voltages. This results in a behavior of the switch that is depending on the voltage amplitude $u_{p}$, which is not desired.

The synthetic impedance consists of two parts, the current amplifier and the admittance transfer function $Y(s)$ which is implemented on a DSpace ds1103 DSP system. According to the SSDI theory, the switch should be triggered precisely at the moments of maximum strain in the first vibration mode. This is realized by an observer, modeled in the DSP system, which estimates the first modal vibration amplitude of the beam. For further details of the test rig, the reader is referred to Neubauer et al. (2011) and Schwarzendahl et al. (2010).
The synthetic impedance realization allows a precise tuning of the network parameters $L$ and $R$. Especially, very low electrical damping $R$ with respect to $\zeta$ can be realized. Figure 11 shows a schematic of the synthetic impedance.

For the measurements, the inductance value was set to $L=3.6817 \mathrm{H}$, which corresponds to an electrical resonance frequency of $200 \mathrm{~Hz}$. This is high enough to ensure a nearly instantaneous charge inversion when the switch is closed. Especially, the influence of the resistance value $R$ with respect to the electrical damping ratio $\zeta$ has been discussed in this article. According to equation (39), the optimal value $\zeta_{\text {opt }}$ is calculated as $4 K / \pi=1.273 \mathrm{~K}$. Therefore, 13 difference resistance values are chosen, so that the damping $\zeta$ covers the range $0.5 K<\zeta<1.5 K$. Figure 12 presents some exemplary time signals of the mechanical vibration amplitude $\eta$ and the voltage $u_{p}$.

The first graphs are performed with an electrical damping ratio smaller than the optimal one, $\zeta<\zeta_{\text {opt }}$. As predicted, a pulsing phenomenon can be observed, due to the oscillation of the energy between the mechanical and electrical system. According to our knowledge, these are the first measurements where such a behavior is observed in the SSDI technique. The smaller the damping ratio $\zeta$, the stronger the pulsing. For the calculated $\zeta_{\text {opt }}$ and for higher values, the vibration amplitudes reduce monotonically.

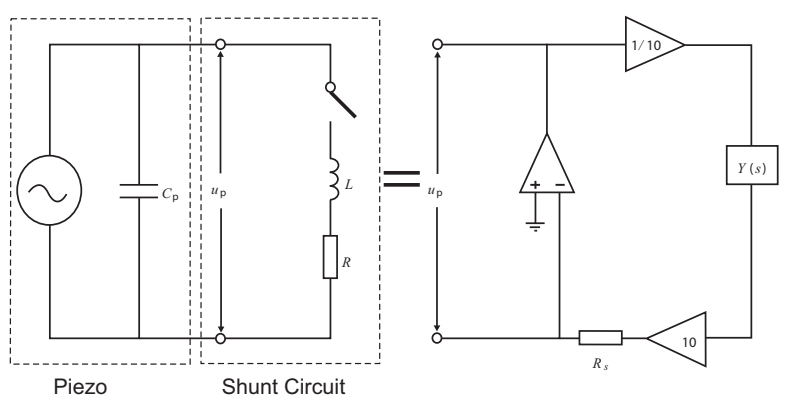

Figure I I. Synthetic impedance circuit. 

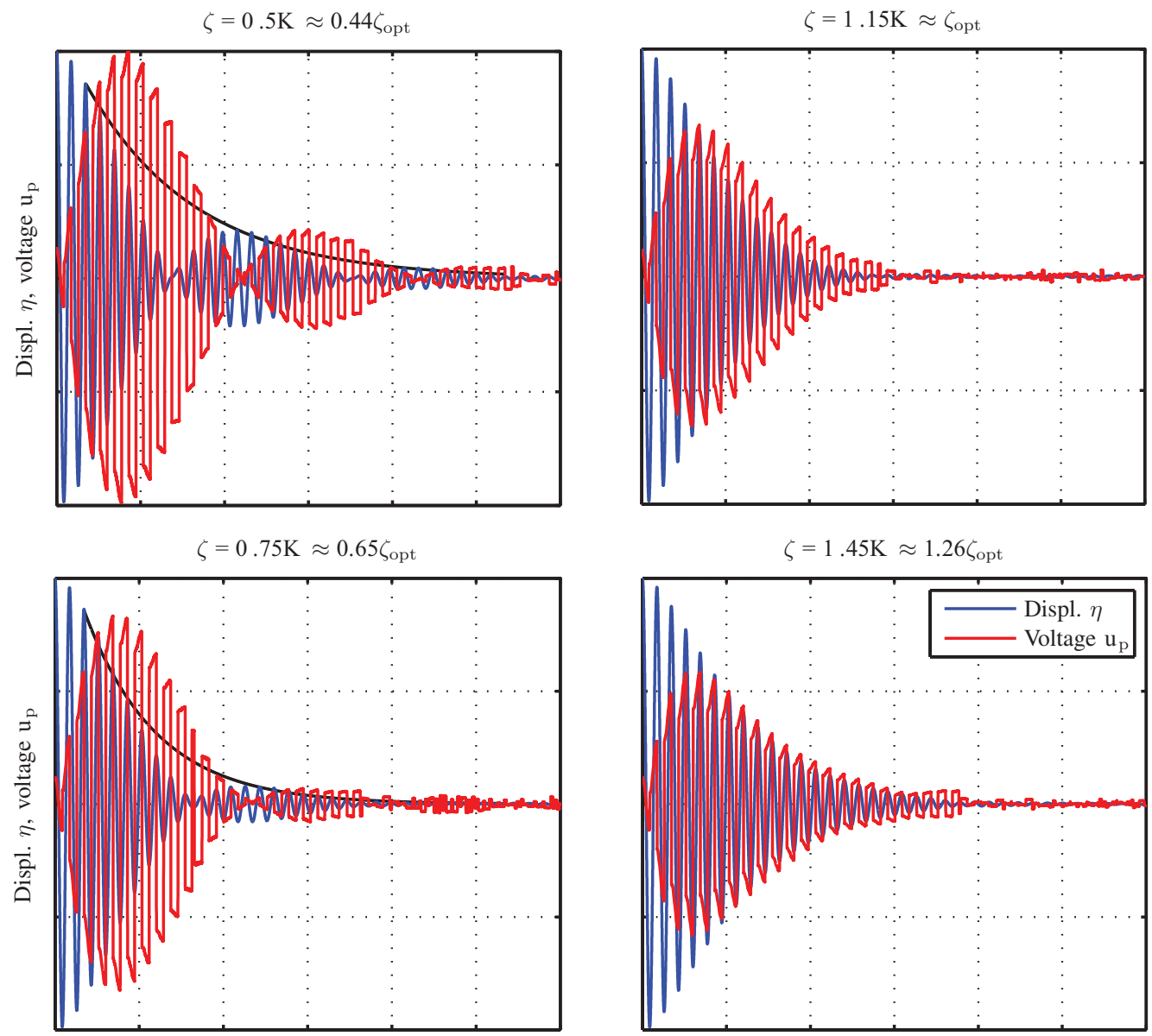

Time $\mathrm{t}$

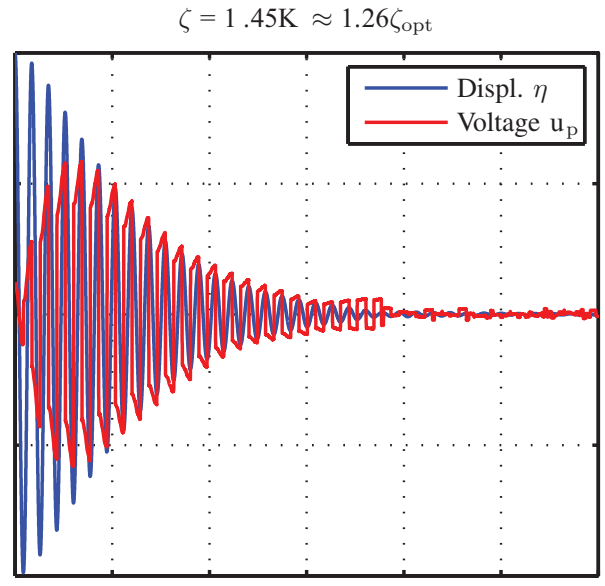

Time $\mathrm{t}$

Figure 12. Exemplary time signals of $\eta$ and $u_{p}$ for four different electrical damping ratios $\zeta$.

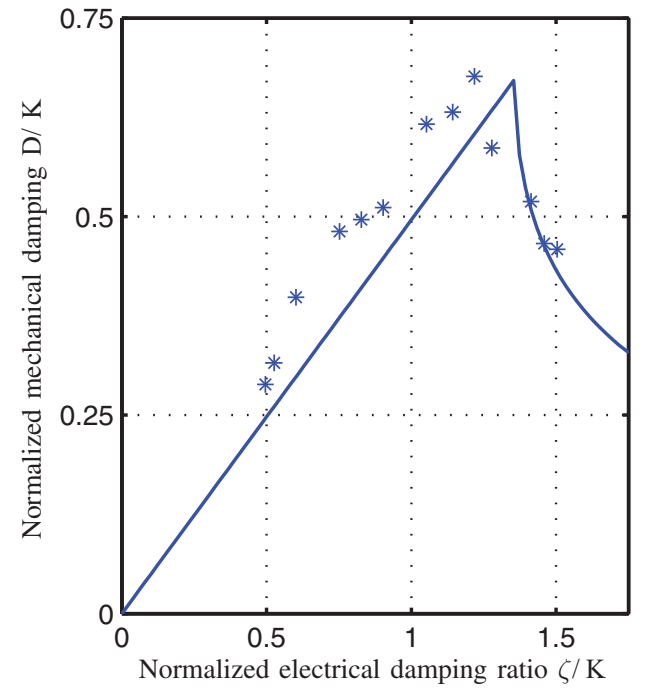

Figure 13. Mechanical damping $D$ versus the electrical damping ratio $\zeta$ : comparison of simulation and measurement.

For each measurement, an envelope of the mechanical vibration amplitudes can be fit, from which the corresponding mechanical damping $D$ is calculated. It can already be seen that the reduction in vibration amplitudes is smaller when the pulsing occurs.

The mechanical damping performance during the measurements is shown in Figure 13 versus the electrical damping ratio $\zeta$.

Each free vibration measurement is represented by one datapoint. For comparison, the calculated damping is included, which is basically the real part of the complex solution given in Figure 8. Generally, the measurements agree very well with the simulation results. There exists an optimal electrical damping ratio $\zeta_{\text {opt }}$; for smaller $\zeta$, the damping performance is reduced due to the pulsing behavior. Generally, the damping in the measurements is slightly higher than the theoretical prediction. One reason for this might be the material damping of the beam, which is neglected in the calculations.

\section{Conclusion}

This article presents the optimal tuning of SSDI circuits for vibration damping of mechanical structures. Based 
on the analytical calculation of the free vibrations, the damping performance is obtained. All calculations are carried out in the most general way, so that the results can be transferred to any mechanical structure. As a result, the optimum electrical damping $\zeta_{\text {opt }}$ and the corresponding maximum damping are determined. These values only depend on the generalized piezoelectric coupling coefficient of the system. Approximations of the exact result clearly show the influence of the generalized coupling coefficient upon the damping performance.

Comparing the damping performance with the resonant $L R$ shunts, the SSDI technique is by a factor 1.27 superior. In both cases, the performance increases linearly with the coupling coefficient $K$. Practically, these results can be used to calculate the optimum resistance by measuring or calculating the coupling $K$.

Measurements are presented to validate the obtained results. A clamped beam with attached piezoceramics is damped by shunting the synthetic impedance at the electrodes. The results are in good agreement with the theoretical results. For the first time, the optimal tuning of the network parameter and the pulsing effect has been obtained in measurements.

\section{Funding}

This research received no specific grant from any funding agency in the public, commercial, or not-for-profit sectors.

\section{References}

Behrens S and Moheimani SOR (2002) Current flowing multiple-mode piezoelectric shunt dampener. In: Smart structures and materials 2002: damping and isolation (ed GS Agnes), San Diego, CA, 18-21 March, Proceedings of SPIE Volume 4697, pp.217-226, Washington, USA.

Clark WW and Schoenly J (2005) Evaluation of performance indices for tuning the switch timing of pulse-switched piezoelectric shunts for vibration control. In: Smart structures and materials 2005: damping and isolation (ed K-W Wang), San Diego, CA, 7-10 March, Proceedings of SPIE Volume 5760, pp.402-412, Washington, USA.

Corr LR and Clark WW (2003) A novel semi-active multimodal vibration control law for a piezoceramic actuator. Journal of Vibration and Acoustics 125(2): 214-222.

Ducarne J, Thomas O and Deu J-F (2010) Structural vibration reduction by switch shunting of piezoelectric elements: modeling and optimization. Journal of Intelligent Material Systems and Structures 21(8): 797-816.

Fleming AJ and Moheimani SOR (2006) Sensorless vibration suppression and scan compensation for piezoelectric tube nanopositioners. IEEE Transactions on Control Systems Technology 14(1): 33-44.

Fleming AJ, Behrens S and Moheimani SOR (2000) Synthetic impedance for implementation of piezoelectric shunt damping circuits. IEE Electronics Letters 36(18): 15251526.

Fleming AJ, Behrens S and Moheimani SOR (2003) Reducing the inductance requirements of piezoelectric shunt damping systems. Smart Materials and Structures 12: 57-64.
Hagood NW and Von Flotow A (1991) Damping of structural vibrations with piezoelectric materials and passive electrical networks. Journal of Sound and Vibration 146: 243-268.

Hollkamp JJ (1994) Multimodal passive vibration suppression with piezoelectric materials and resonant shunts. Journal of Intelligent Material Systems and Structures 5: 49-57.

Lefeuvre E, Badel A, Petit L, et al. (2006) Semi-passive piezoelectric structural damping by synchronized switching on voltage sources. Journal of Intelligent Material Systems and Structures 17(8-9): 653-660.

Moheimani SOR (2003) A survey of recent innovations in vibration damping and control using shunted piezoelectric transducer. IEEE Transactions on Control Systems Technology 11(4): 482-494.

Neubauer M and Wallaschek J (2008) Analytical and experimental investigation of the frequency ratio and switching law for piezoelectric switching techniques. Smart Materials and Structures 17(3): 035003 (9pp.).

Neubauer M and Wallaschek J (2010) Vibration damping with shunted piezoceramics: fundamentals and technical applications. In: 6th international conference mechatronics systems and materials (MSM), Opole, Poland, 5-8 July.

Neubauer M, Han X and Schwarzendahl SM (2011) Enhanced switching law for synchronized switch damping on inductor with bimodal excitation. Journal of Sound and Vibration 330(12): 2707-2720.

Neubauer M, Niederberger D and Morari M (2006) A novel approach for brake squeal control using shunted piezoceramics. In: Proceedings of 24th SAE brake colloquium and exhibition, Grapevine, TX, 8-11 October.

Neubauer M, Oleskiewicz R, Popp K, et al. (2006) Optimization of damping and absorbing performance of shunted piezo elements utilizing negative capacitance. Journal of Sound and Vibration 298: 84-107.

Niederberger D and Morari M (2006) An autonomous shunt circuit for vibration damping. Smart Materials and Structures 15: 359-364.

Niederberger D, Fleming A, Moheimani SOR, et al. (2004) Adaptive multi-mode resonant piezoelectric shunt damping. Smart Materials and Structures 13: 1025-1035.

Preumont A (2006) Mechatronics: Dynamics of Electromechanical and Piezoelectric Systems. Springer, The Netherlands.

Richard C, Guyomar D, Audigier D, et al. (2000) Enhanced semi-passive damping using continuous switching of a piezoelectric device on an inductor. In: Smart structures and materials 2000: damping and isolation (ed TT Hyde), Newport Beach, CA, 6 March, Proceedings of SPIE Volume 3989, pp.288-299, Washington, USA.

Schwarzendahl SM, Han X, Neubauer M, et al. (2010) Optimized switching algorithm for synchronized switch damping for multimodal excitation. In: Active and passive smart structures and integrated systems 2010, San Diego, CA, 8 March, Proceedings of SPIE Volume 7643, Washington, USA.

Tang J and Wang KW (2001) Active-passive hybrid piezoelectric networks for vibration control: comparisons and improvement. Smart Materials and Structures 10: 794-806.

Wu S-Y (1998) Method for multiple-mode shunt damping of structural vibration using a single PZT transducer. In: Smart structures and materials 1998: passive damping and isolation (ed LP Davis), San Diego, CA, 2 March, Proceedings of SPIE Volume 3327, pp.159-168, Washington, USA. 